\title{
Tumor Cell Detection by Mass Spectrometry using Signal Ion Emission Reactive Release Amplification (SIERRA)
}

Zane Baird†,§, Valentina Pirro§, Stephen Ayrton§, Adam Hollerbach§, Cathleen Hanau†, Karen Marfurt†, Mary Foltz†, R. Graham Cooks§, ${ }^{*}$ and Michael Pugia†, ${ }^{*}$

†Siemens Healthcare Diagnostics, 3400 Middlebury Street, Elkhart, IN, USA.

§Chemistry Department, Purdue University, 560 Oval Drive, West Lafayette, IN, USA.

Corresponding Authors

*Email: cooks@purdue.edu; michael.pugia@siemens.com

\section{Electronic Supporting Information}

\section{Table of Content}

1. Functionalization of silica nanoparticles (SiNP) with IC9-1 and IC9-2 $\ldots \ldots \ldots \ldots \ldots \ldots \ldots \ldots \ldots \ldots \ldots \ldots \ldots \ldots \ldots \ldots \ldots \ldots \ldots$

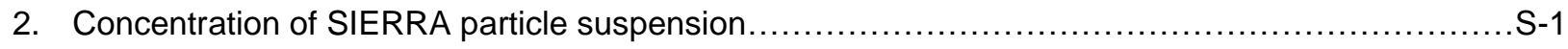

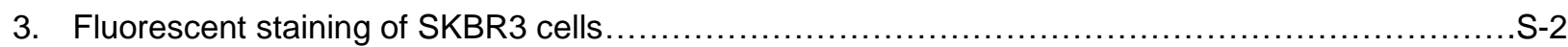

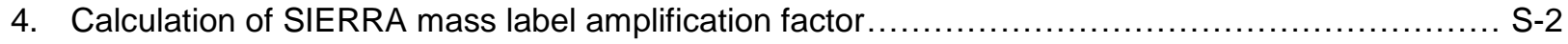

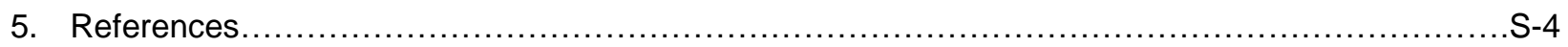




\section{Functionalization of silica nanoparticles (SiNP) with IC9-1 and IC9-2}

A solution of $7.5 \mathrm{mg}$ of $\mathrm{N}$-succinimidyl 3-(2-pyridyldithio)propionate (SPDP) (in $1.6 \mathrm{~mL}$ dimethyl sulfoxide (DMSO) was prepared, and $75 \mu \mathrm{L}$ of this solution was added to $1 \mathrm{~mL}$ of phosphate buffered saline (PBS) containing $10 \mathrm{mg}$ of propylamine functionalized SiNP in a $1.5 \mathrm{~mL}$ microcentrifuge tube. The mixture was mixed on a roller mixer at 75 RPM for 30 min at room temperature and then centrifuged at 2000 RPM for $3 \mathrm{~min}$. The supernatant was removed, the particles were resuspended in $1 \mathrm{~mL}$ PBS, and the suspension was sonicated for $10 \mathrm{~s}$ at $20 \%$ amplitude, $1 \mathrm{~s}$ on, $3 \mathrm{~s}$ off with a microtip probe on a Q500 sonicator (Qsonic, Newtown, CT). The centrifugation, supernatant removal, resuspension, and sonication procedure was repeated twice more to thoroughly wash the particles. The particles were once again centrifuged, the supernatant removed, and particles resuspended in a solution of $8.0 \mathrm{mg} \mathrm{IC9-1}$ and 1.0 mg IC9-2 in $1 \mathrm{~mL}$ PBS. This suspension was mixed overnight at room temperature on a roller mixer at 75 RPM. The resulting suspension of IC9-1/IC9-2 functionalized SiNP was then washed twice more (centrifuged, decanted, resuspended in $1 \mathrm{~mL}$ PBS, and sonicated). The final suspension of IC9-1/IC9-2 functionalized SiNP was then stored at $4{ }^{\circ} \mathrm{C}$.

\section{Concentration of SIERRA particle suspension}

The concentration of magnetic particles in the suspension of the original Sera-mag beads can be calculated from the properties of the magnetic particles as given by the manufacturer (shown in Table 1S).

Table 1S. Properties of Sera-Mag magnetic beads

\begin{tabular}{lll}
\hline Property & Units & Value \\
\hline Density $(\boldsymbol{\rho})$ & $\mathrm{g} / \mathrm{cm}^{3}$ & 1.7 \\
\hline Radius $(\boldsymbol{r})$ & $\mu \mathrm{m}$ & 0.378 \\
\hline Concentration $(\boldsymbol{C})$ & $\mathrm{mg} / \mathrm{mL}$ & 10 \\
\hline
\end{tabular}

$$
\frac{\text { Particles }}{m L}=\frac{C}{\rho \times 1000\left[\frac{4}{3} \pi\left(r \times 10^{-4}\right)^{3}\right]}=2.60 \times 10^{10}
$$

or the sake of simplicity, it is assumed that no magnetic particles are lost in the preparation of the final SIERRA particles. With this assumption the concentration of SIERRA particles in the stock solution is calculated as:

$$
\frac{(0.104 \mathrm{~mL})\left(2.60 \times 10^{10} \text { particles } / \mathrm{mL}\right)}{1 \mathrm{~mL}}=2.70 \times 10^{9} \text { particles } / \mathrm{mL}
$$




\section{Fluorescent staining of SKBR3 cells}

A solution of approximately $10^{5}$ SKBR3 cells stored in $500 \mu \mathrm{L}$ of medium buffer were centrifuged for 2 min at $2500 \mathrm{~g}$ (all centrifugation for cell staining was performed with the same settings), after which the supernatant was decanted and discarded, leaving behind a cell pellet. Cells were washed with $1.0 \mathrm{~mL}$ of PBS by gentle vortexing followed by centrifugation. Cells were washed with $0.2 \%$ TritonX in PBS (1.0 $\mathrm{mL}$ ), permeabilized for $7 \mathrm{~min}$ with another aliquot of the same solution, washed four times with PBS (1.0 $\mathrm{mL}$ each), blocked with $1.0 \mathrm{~mL}$ of casein buffer (Candor Bioscience $\mathrm{GmbH}$, Wangen, Germany), and washed twice with $1.0 \mathrm{~mL}$ of $0.05 \%$ Tween in PBS. The blocking solution was added to prevent nonspecific binding of antibodies. A $10 \mu \mathrm{g} / \mathrm{mL}$ solution of the CK8/18-Dyl550-biotin antibody was prepared in casein buffer from the $1.0 \mathrm{mg} / \mathrm{mL}$ stock solution. $300 \mu \mathrm{L}$ of the dilute solution was added to the tube containing the cells and the mixture was incubated at room temperature on a rotary mixer (75 RPM) for $25 \mathrm{~min}$. This mixture was then centrifuged, the supernatant removed, and the cells washed 4 more times with $1 \mathrm{~mL}$ of PBS with $0.05 \%$ Tween. Following the final wash, the supernatant was removed, and $500 \mu \mathrm{L}$ of a $1 \mu \mathrm{g} / \mathrm{mL}$ 4,6-diamidino-2-phenylindole (DAPI, Thermo Scientific) solution in PBS was added. The mixture was gently vortexed and allowed to react for 1 minute. The cells were then washed with 3 separate $1 \mathrm{~mL}$ aliquots of PBS with $0.05 \%$ Tween. After the final wash, the cells were resuspended in 1.2 $\mathrm{mL}$ of PBS, and $2.0 \mu \mathrm{L}$ of this solution was spotted on a slide and examined by fluorescent microscopy to determine a final cell count of $\sim 1.2 \times 10^{4}$ cells $/ \mathrm{mL}$.

\section{Calculation of SIERRA mass label amplification factor}

In order to calculate the maximum amplification factor that could be achieved with the SIERRA method, several assumptions were made. First, the theoretical maximum number of nanoparticles that could be bound to a streptavidin coated magnetic bead was calculated. This was done by assuming that the nanoparticles formed a close-packed, concentric shell around the spherical magnetic bead, a crosssectional view of which is shown in Figure $1 \mathrm{~S}$ below. 


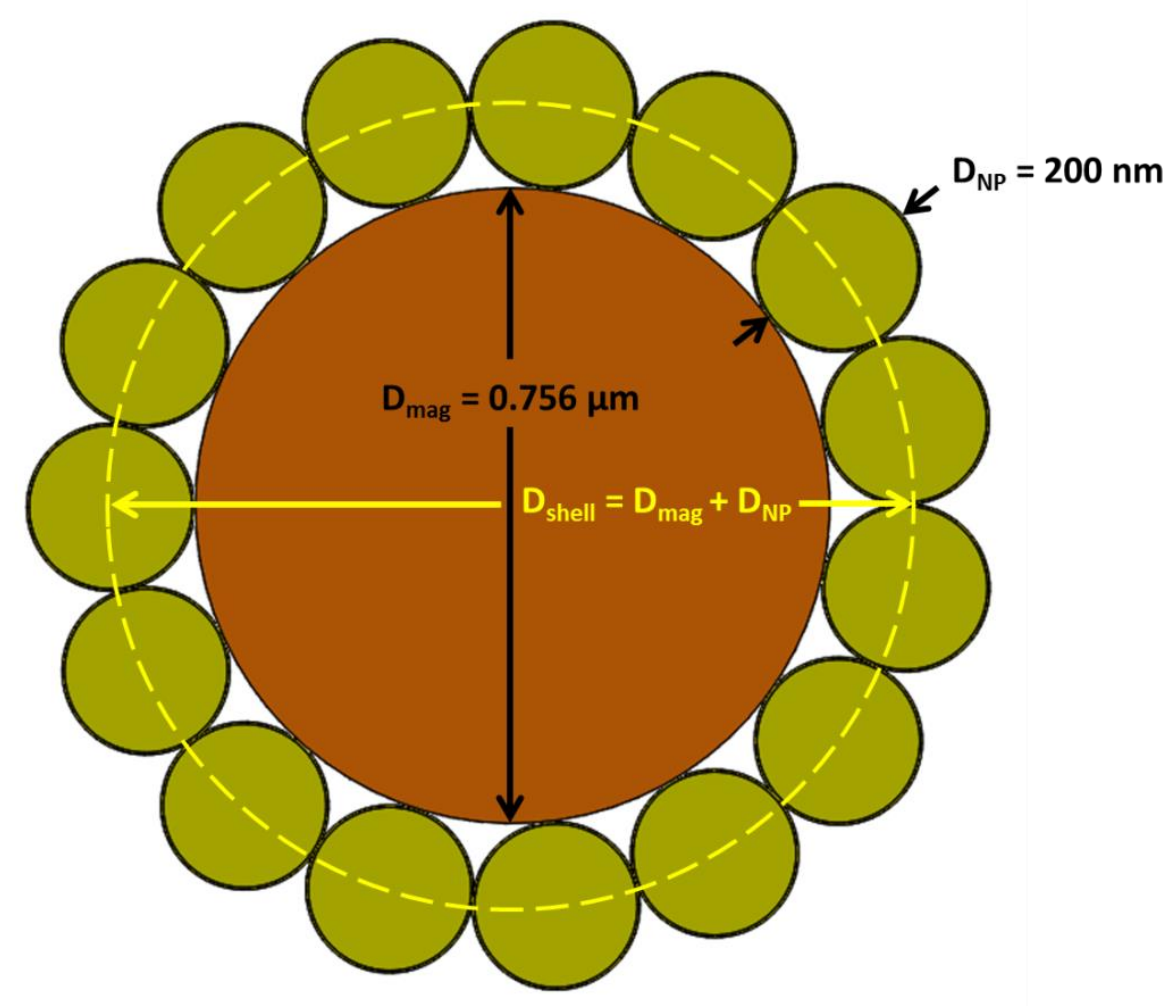

Figure 1S. Cutaway view of nanoparticle (yellow) packing around magnetic bead (orange). The yellow inscribed circle is used to calculate the close-packing of disks on the spherical shell for an estimation of 3Dimensional packing of nanoparticles around a sphere. Drawing is shown to-scale.

The problem was further simplified by considering the packing of circles on a spherical surface that defined by the inscribed yellow circle shown in Figure 1S. The surface area of this sphere $\left(S_{\text {shell }}\right)$ was calculated as follows:

$$
S_{\text {shell }}=4 \pi\left(\frac{D_{\text {shell }}}{2}\right)^{2}=2.871 \mu m^{2}
$$

According to a numerical study from B. W. Clare et. al regarding the packing of circles within a spherical surface, packing numbers greater than 40 typically result in surface coverage with an average of $\sim 0.815^{1}$. Since it is reasonable to assume that packing numbers in excess of 40 are expected within the context of this work, a packing efficiency of 0.815 was used to estimate the maximum number of SiNP $\left(N_{\max }\right)$ that may be packed around a single magnetic bead. This was calculated from the surface area of a circle with a diameter equal to that of the $\operatorname{SiNP}\left(D_{N P}, 200 \mathrm{~nm}\right)$ and the previously calculated $S_{\text {shell. }}$

$$
N_{\text {max }}=0.815\left(\frac{S_{\text {shell }}}{\pi\left(\frac{D_{N P}}{2}\right)^{2}}\right)=74 N P S
$$

Following the same calculation and merely considering the relative size between a cancerous cell and the magnetic particles, the maximum number of magnetic particles that can be packed around a cell is estimated to be approximately 730 . 
It was also necessary to determine the amount of IC9-1 which may be bound to a single SiNP. Because little information is provided by the manufacturer of the mesoporous SiNP, a number of assumptions were made based on the relative properties of known and well-characterized mesoporous silica. A common form of mesoporous silica is termed MCM-41, the bulk properties of which are provided by SigmaAldrich ${ }^{2}$. From the particle diameter and bulk density $\left(\rho_{N P}\right)$ of $0.34 \mathrm{~g} / \mathrm{mL}$, the mass of a single particle $\left(M_{N P}\right)$ was estimated as follows:

$$
M_{N P}=\rho_{N P}\left[\frac{4}{3} \pi\left(\frac{D_{N P}}{2}\right)^{3}\right]=1.424 \times 10^{-15} g
$$

According to analysis of amine-functionalized MCM-14 performed by López-Aranguren et al, this material was functionalized with as much as $3.99 \mathrm{mmol} / \mathrm{g}$ amine groups. The maximum number of available sites for peptide modification was calculated as the total number of amine groups per $\operatorname{SiNP}\left(N_{\text {peptide }}\right)$ :

$$
N_{\text {peptide }}=M_{N P}\left(3.99 \times 10^{-3} \text { mole } / g\right)\left(6.022 \times 10^{23} \text { molecules } / \text { mole }\right)=3.42 \times 10^{6} \text { peptides } / N P
$$

Because the peptide mass label (IC9-1) only constitutes for 0.906 of the total molar loading (8.0 mg IC9$1,1.0 \mathrm{mg} \mathrm{IC9}-2)$ on each SiNP, this must was factored into the final amplification factor provided by a single SIERRA particle $\left(X_{\text {SIERRA }}\right)$ :

$$
X_{\text {SIERRA }}=0.906 \times N_{\text {max }} \times N_{\text {peptide }}=2.29 \times 10^{8}
$$

Microscopic examination of the isolated cells that had been incubated with fluorescein labeled SIERRA particles suggest that more than 100 particles may be present on a single cell, giving a final amplification of $>2.29 \times 10^{10}$ mass labels per cell. It is important to note that cytokeratins 8 and 18 may show varying levels of expression among cells, which combined with size variation of SKBR3 cells makes this number only a very rough estimate of the amplification possible by the SIERRA method.

\section{References}

B. W. Clare, D. L. Kepert, J. Math. Chem., 1991, 6, 325

Sigma-Aldrich, http://www.sigmaaldrich.com/catalog/product/aldrich/643645?lang=en\&region=US, (accessed April 2016) 